\title{
Influence of Lithium Salt in Polymer Electrolytes on Solid-Electrolyte Interphase (SEI) Characterized by Cryogenic-Transmission Electron Microscopy
}

Vahid Jabbari ${ }^{1}$, Yifei Yuan ${ }^{2}$, Kun $\mathrm{He}^{3}$ and Reza Shahbazian-Yassar ${ }^{1}$

${ }^{1}$ University of Illinois at Chicago, Chicago, Illinois, United States, ${ }^{2}$ University of Illinois at Chicago, United States, ${ }^{3}$ Northwestern University, United States

Rechargeable lithium-metal batteries (LMBs) are considered one of the most promising candidates among high-energy-density energy storage technologies for portable electronics and electric vehicles. However, dendritic growth of lithium induces poor cycling performance, low Coulombic efficiency, and safety risk (e.g. short-circuit and thermal runaway), hindering practical applications of LMBs [1]. Formation and growth of Li dendrite is closely related to the insoluble deposit layer-called solid-electrolyte interphase (SEI) - formed on the Li metal surface. Indeed, SEI layer acts as barrier layer to prevent further direct physical contact between the electrode and electrolyte, thus assuring stability of Li metal and hindering decomposition of electrolyte. Thus, an ideal SEI should own several important features including maximum Li-ion conductivity while having a minimum electrical conductivity, a compact structure with a proper thickness, high elastic modulus to mechanically hinder the Li dendrite breakthrough. Therefore, fundamental understanding of SEI in LMBs is considered the first step to make efficient LMBs with longterm cycle life [2]. Generally, SEI has a multilayer-type or mosaic-type structure, composed of inorganic and organic/polymeric species. Transmission electron microscopy (TEM) is a unique and powerful technique which enables direct observation of the SEI - the formation and evolution - and identification of nature of the structure and composition. Considering the beam-sensitivity of soft Li metal or SEI, they are unstable under electron beam radiation of conventional TEM. In recent years, cryogenic-transmission electron microscopy (cryo-TEM) is used to analyze battery materials including SEI. Cryogenic condition preserves the intrinsic structure/composition of the sample while mitigating the beam damage issue. Combining electron energy loss spectroscopy (EELS) and energy-dispersive X-ray spectroscopy (EDS), cryo-TEM can offer new insights into the structure and composition of Li metal anode and the SEI layer [3]. Herein, we aim to identify structure and composition of the SEI layer in LMBs derived from solid polymer electrolytes (SPEs) made of PEO and different Li salts (LiTFSI or LiPF6). This study can offer new insights into the influence of type of Li salts on the cycle life and efficiency of solid state LMBs. It is known that PEO-LiTFSI SPE exhibits a superior electrochemical performance compared to PEO-LiPF6 SPE. This is ascribed to less stable nature of LiPF6 generate HF and PF5 gases in contact with solvent. For instance, it is shown that LiPF6 is less stable and generate HF and PF5 gases in contact with solvent 
which can pose detrimental impact on the LMBs performance. Yet, detailed structure/composition of the Li metal anode-SPE interface and the SEI layer is unknown. Hence, this study deals with identification of nature of the SEI layer formed using different Li salts in polymer electrolyte by cryo-TEM. EDS and EELS spectroscopies are also used to identify the chemical nature of the SEI layer. Cryo-TEM analysis of the electrochemically plated Li particles using P(EO)15-LiTFSI SPE is shown in Figure 1. A mosaictype SEI layer is observed which is made of inorganic Li species dispersed in an amorphous organic (polymeric) matrix.

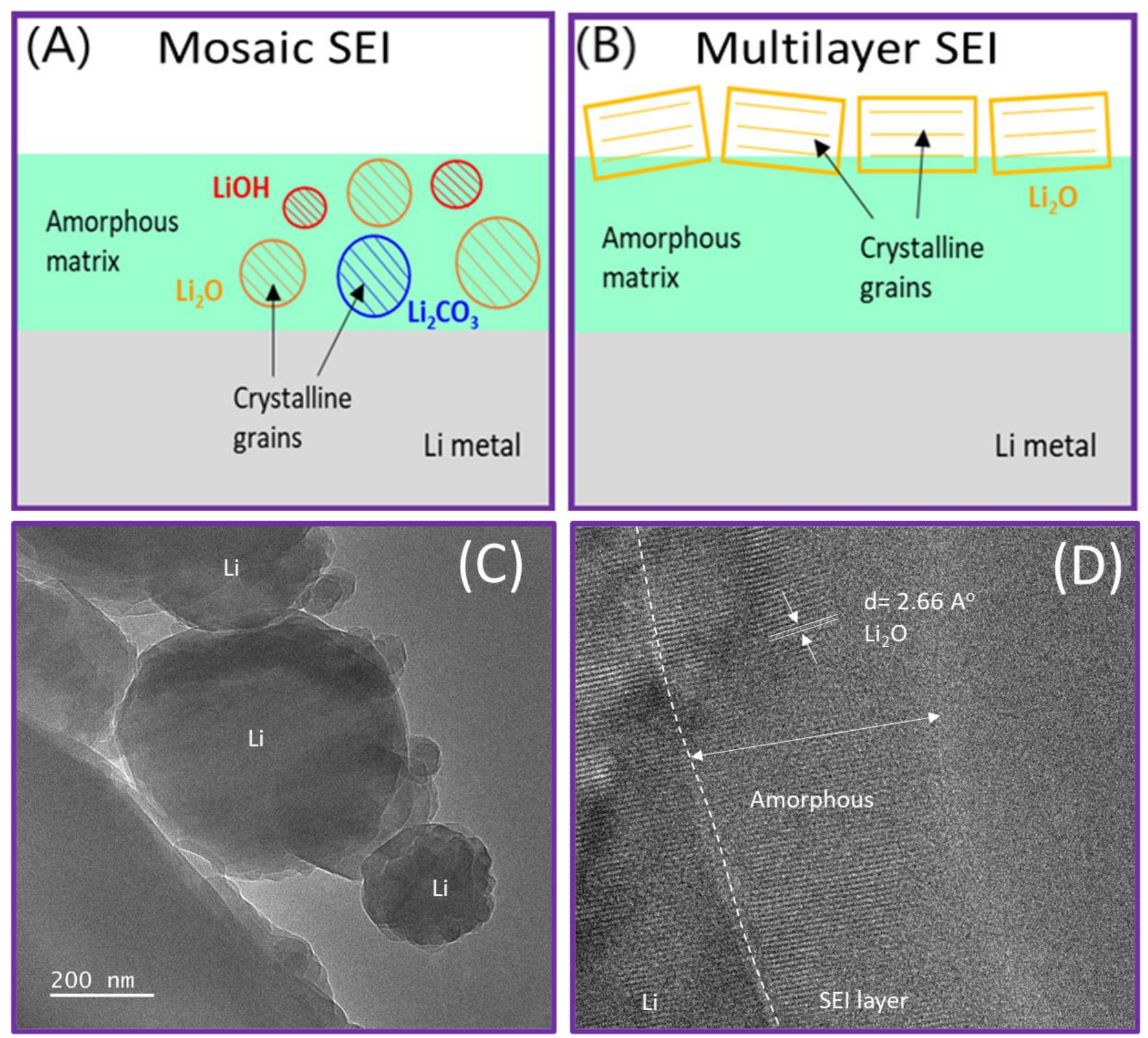

Figure 1. Figure 1. Cryo-TEM analysis of the SEI layer. Schematic illustration of mosaic (A) and multilayer (B) SEI. Cryo-TEM image of electrochemically deposited Li particles in Li/Cu cell made with PEO- 
LiTFSI SPE (C) and the surface layer (D). A distinct SEI layer is shown on the Li particle which is made of Li species dispersed into an amorphous matrix.

\section{References}

[1] S. Lou, Z. Yu,1 Q. Liu, H. Wang, M. Chen, J. Wang, Chem 2020, 6, 1-20.

[2] A. Wang, S. Kadam, H. Li, S. Shi, Y. Qi, npj Computational Materials 2018, 4, 15.

[3] M.J. Zachman, Z. Tu, S. Choudhury, L.A. Archer, L.F. Kourkoutis, Nature 2018, 560, 345-349. 